\title{
GENERATING SIMULATION NETWORK FOR DESIGN PROCESS BASED ON DSM
}

\author{
M. A. Hossain \\ D. K. H. Chua \\ 1 Engineering Drive 2 \\ E1A-03-07 \\ Dept. of Civil Engineering \\ SINGAPORE 117576
}

\begin{abstract}
Use of simulation technique is becoming more popular in managing design process. However, developing a simulation model can be very tedious and knowledge demanding, and a simulation model developed for one project cannot readily be used for others due to the unique nature of design project. This paper develops a generalized simulation model for the design process which is capable of generating simulation network automatically for any design project based on dependency relationships as represented by DSM. Concept of estimability and rework of design activities has been utilized in reducing the overall design completion time. Simulation can be run for the generated network to get a probabilistic design schedule. The proposed concept of autogeneration of simulation network has been studied with a case study. It is very efficient in generating the simulation network for any design process and saves the tedious job of simulation model development.
\end{abstract}

\section{INTRODUCTION}

Simulation technique has been found to be effective in scheduling design process and gives a prior confidence before implementing it. Simulation deals more efficiently with the stochastic and random nature of activity durations and provides more versatile analytical capabilities (Lee and Arditi 2006). However, field application of simulation for the design project in construction industry is limited. As stated in Chua and Li (2002) and Kim and Gibson (2003), developing a simulation model is complex and considerable amount of time and cost are involved. Moreover, design projects are unique in nature and not repetitive because they are multi-disciplinary; various activities, parties, and resources are involved in a single project; and their dependencies are also different. So, simulation model developed for one project cannot be readily applied to other project.

Some general purpose simulation techniques and languages are also available in the field of AEC from last few decades such as CYCLONE (Halpin 1976), UM-CYCLONE (Ioannou 1990), CIPROS (Odeh 1992), COOPS (Liu and Ioannou 1992) STROBOSCOPE (Martinez 1996) and so on. However, scheduling design processes using the aforementioned simulation languages is highly complex and tedious. Expert knowledge on simulation technique is needed for developing simulation model. In an attempt to simplify the simulation modeling procedure, RISim (Chua and Li 2001, 2002) presents a resourceinteracted simulation modeling method. In RISim, a construction project is conceived as a collection of resources involved and their interaction; and resources may have their own process within themselves. However, this model is not generalized and restricted to construction operations modeling. Ioannou and Martinez (1996) describe about scalable simulation models which can facilitate auto code generation of network for repetitive construction operations; for example, modeling a vertical transportation of construction labor in a high-rise building construction. Queuing behavior for labors waiting to go up or down are similar for each floor though the number of labor may be different. So, in this case, autogeneration is done for same nodes and links for each floor. Nevertheless, due to unique nature of design project in terms of different attributes of design activities and diverse dependencies between these activities, traditional practice is to develop a simulation model for each project which is expensive and time consuming.

Autogeneration of simulation network would be worthwhile in saving time and cost for a project. This paper describes a model which is able to automatically generate simulation network for scheduling the design process based on design structure matrix (DSM). The model incorporates the concept of utilizing early estimated information while accounting for rework which is typical of the industry. Autogeneration will save the tedious job of developing simulation network and is useful for those who have little knowledge in simulation techniques. Effectiveness of the model has been elaborated with a case study. 


\section{Hossain and Chua}

2

\section{CONCEPT OF ESTIMABILITY AND REWORK IN DESIGN PROCESS}

Design activities are listed in rows and columns sequentially in the same order in DSM (see Table 1) to represent the dependencies between activities (Steward 1981, Austin et al. 2000). In the DSM, each ' 1 ' indicates that corresponding row activity is dependent on the activity above it and ' 0 ' indicates that there is no such relationship. Although, DSM can produce an appropriate order of design activities to account for information dependency and minimize feedback loops, traditionally design schedule strictly follows this order by waiting for confirmed parameters from preceding activities and lengthen design completion time. This scenario is depicted in Figure 1(a) which shows the traditional finish-start dependency. In contrast, if parameter/information from $A_{1}$ is estimable then $A_{2}$ can start earlier instead of waiting for the full analysis of $A_{1}$ as depicted in Figure 1(b). Similarly, $A_{3}$ can start earlier if both $A_{1}$ and $A_{2}$ are estimable and so on. But the later is accompanied by the possibility of rework in downstream activities if values of the parameters deviate from the assumed design range when the parameters are eventually finalized. In general, the parameters utilized in the full analysis are in a combination of states (estimated, full analysis, or confirmed) depending on the status of the predecessor activities. Chua and Hossain (2008) showed how using early estimated parameter/information can reduce overall design completion accounting the time required for rework.

Table 1: Activity dependency with DSM

\begin{tabular}{|cccccccc|}
\hline Activity & $\mathbf{1}$ & $\mathbf{2}$ & $\mathbf{3}$ & $\mathbf{4}$ & $\mathbf{5}$ & $\mathbf{6}$ & $\mathbf{7}$ \\
$\mathbf{1}$ & 0 & 0 & 0 & 0 & 0 & 0 & 0 \\
$\mathbf{2}$ & 1 & 0 & 0 & 0 & 0 & 0 & 0 \\
$\mathbf{3}$ & 1 & 1 & 0 & 0 & 0 & 0 & 0 \\
$\mathbf{4}$ & 1 & 0 & 1 & 0 & 1 & 1 & 0 \\
$\mathbf{5}$ & 0 & 1 & 0 & 1 & 0 & 0 & 0 \\
$\mathbf{6}$ & 0 & 0 & 1 & 1 & 0 & 0 & 0 \\
$\mathbf{7}$ & 0 & 1 & 0 & 0 & 1 & 1 & 0 \\
\hline
\end{tabular}
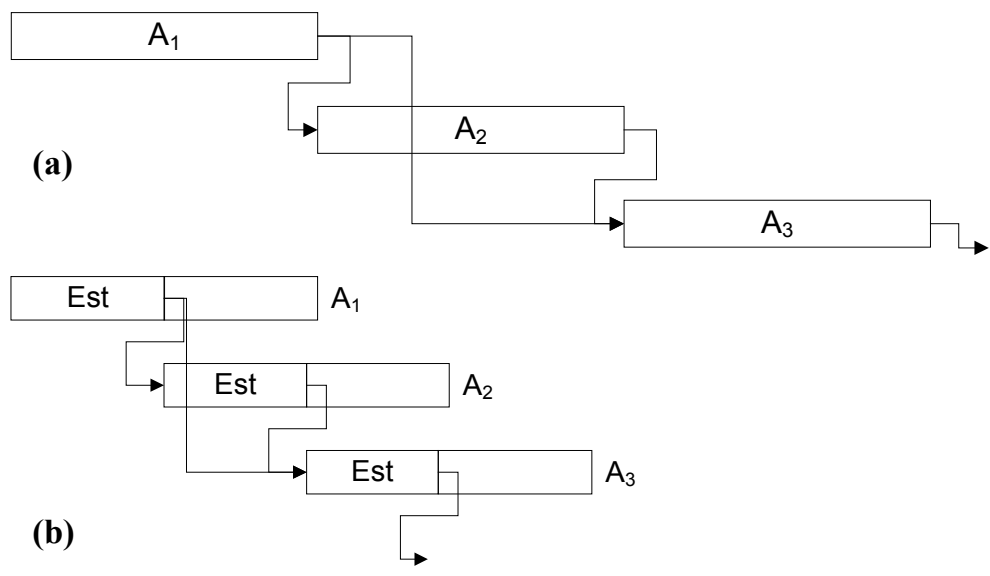

Figure 1: (a) Traditional finish-start dependency, (b) Early information sharing with estimation

Figure 2 depicts the simulation model (developed with STROBOSCOPE) incorporating the concept of early estimation and rework. As can be seen from Table 1, activity $A_{3}$ is dependent on parameter from $A_{1}$ and $A_{2}$ and it transmits parameter to activity $A_{4}$ and $A_{6}$. The Figure shows design process only for $A_{3}$ with the relevant dependencies, which is only a part of the whole design process. Full analysis of $\mathrm{A}_{3}$ (shaded Combi) requires parameters from $\mathrm{A}_{1}$ and $\mathrm{A}_{2}$ and can be drawn through the Queues 'DataReady $A_{1} T o A_{3}$ ' and 'DataReady $A_{2} T o A_{3}$ ' respectively. Required parameter from $\mathrm{A}_{1}$ can be obtained from one of two ways: the full analysis via Combi 'FullAnalysis $A_{1}$ ' or early estimation via 'Estimate $A_{l}$ '. On the other hand, parameter from $\mathrm{A}_{2}$ can come from three sources: early estimation through 'Estimate $A_{2}$ ', full analysis through 'FullAnalysis $A_{2}$ ' and confirmed value after checking for rework. The check is necessary because activity $\mathrm{A}_{2}$ itself depends on $\mathrm{A}_{1}$ which could have been estimated when the analysis was performed. 


\section{Hossain and Chua}

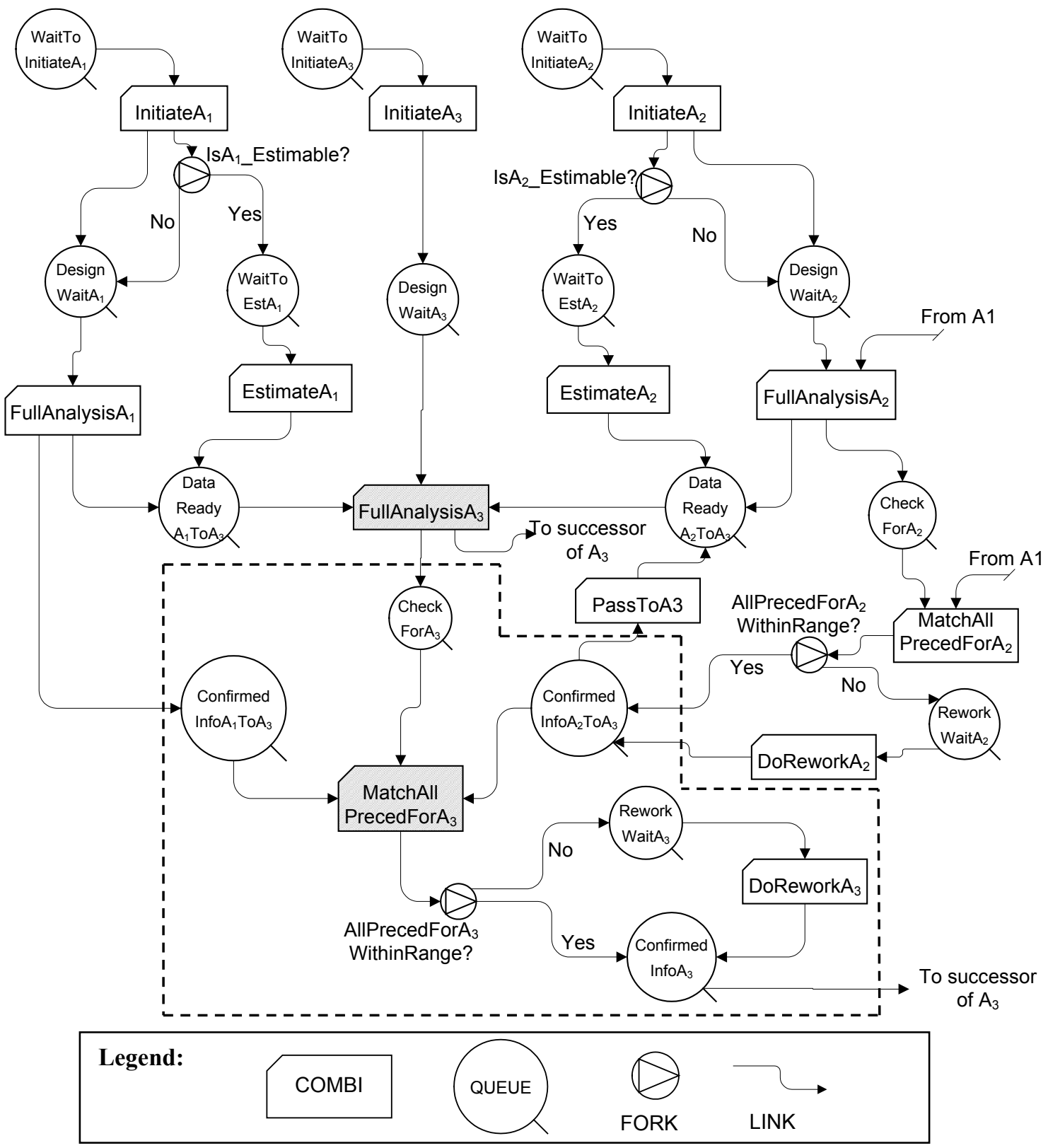

Figure 2: Simulation model with the concept of estimability and rework

As depicted in the Figure, since activity $\mathrm{A}_{1}$ and $\mathrm{A}_{2}$ are the predecessors of $\mathrm{A}_{3}$, relevant queues 'DataReady $A_{1}$ ToA ${ }_{3}$ ' and 'DataReady $\mathrm{A}_{2} \mathrm{ToA}_{3}$ ' are joined with the Combi 'FullAnalysis $\mathrm{A}_{3}$ ' to transmit parameter to $\mathrm{A}_{3}$. Table 1 shows that activity $\mathrm{A}_{1}$ is the predecessor for $\mathrm{A}_{2}$ and $\mathrm{A}_{4}$ as well. So, similar Queues 'DataReadyA $A_{1} T o A_{2}$ ' and 'DataReadyA $A_{1} T o A_{4}$ ' should be created to transmit parameter to those activities. The same is true for other dependent nodes such as 'ConfirmedInfoA ${ }_{2} \mathrm{ToA}_{3}$ '. Taking into account these issues, design process for other activities can be modeled with appropriate dependencies. It is inevitable that developing the simulation network for the whole design process will be very tedious and will be very complicated with the increasing number of design activities and dependencies which is typical in most deign projects. Several thousand of lines of coding will be needed to define the simulation network. The following section describes how a simulation network can be generalized for various activities and the whole simulation network can be generated automatically based on DSM. 


\section{Hossain and Chua}

\section{3}

GENERALIZED SIMULATION NETWORK FOR DESIGN PROCESS

To autogenerate simulation network for any design project, a generalized approach should be adopted. Figure 3 describes the flow chart of generalized approach to generate simulation network for the design process. Firstly, design activities are put into a DSM with dependency relationships. Based on dependency relationships, activities can be categorized into three types and will be elaborated later in this section. After that, generalized internal process for each type of design activities are defined based on how information is transmitted to its successor, though each activity has its own way of analysis. Lastly, based on DSM, specific network is generated using the internal design process.

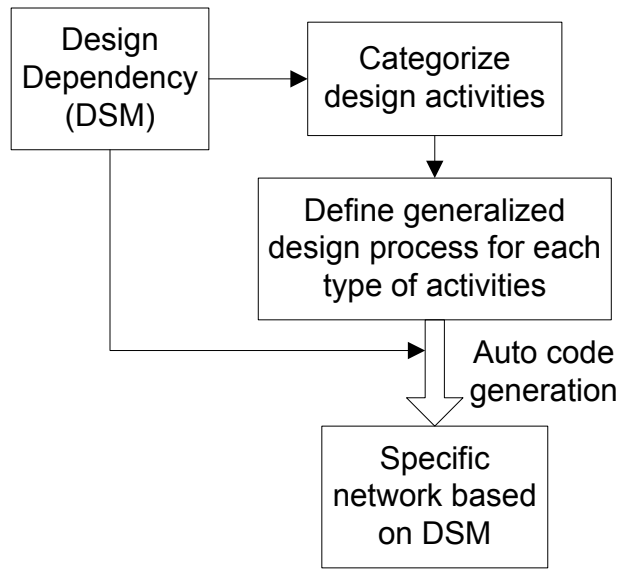

Figure 3: Generalized approach to autogenerate the simulation network

Following the generalized approach, Figure 4 depicts the generalized simulation network for design process. Firstly, based on dependency relationships as represented by DSM, some activities are independent to start its full analysis depicting that this type of activities does not have any predecessor(s) but usually have successor(s). Design process of this type of activities is presented with subscript ' $i$ ' in the Figure (left portion, outside the dashed box). Since i-type activities are independent, design process finishes with full analysis (depicted by the shaded Combi 'FullAnalysis $A_{n}^{i}$ ') and check for rework is unnecessary. The notation $\mathrm{A}_{\mathrm{n}}^{\mathrm{i}}$ describes that it is an ' $\mathrm{i}$ ' type activity and ' $\mathrm{n}$ ' stands for the unique activity ID or activity number. While, this type of activities has successor and it is checked whether the activity is estimable or not through the Queue 'IS $A_{n}^{i}$ Estimable?'. If the activity is estimable then estimation is done and preliminary parameter is transmitted to its successors for greater concurrency. Otherwise, it does its full analysis and transmits confirmed parameter.

Secondly, most of the design activities have both predecessors and successors. Design process for this type of activities is represented by subscript ' $j$ ', as depicted by two dashed boxes in the Figure. The upper dashed box depicts full analysis and the lower dashed box shows the rework process. Full analysis of j-type activity is similar to the design process for i-type activity except the Combi 'FullAnalysis $A_{n}^{j}$ ' is constrained by the predecessor parameter through the Queue 'DataReady $A_{x \text { - }}^{i}$ $T o A_{y}^{j}$ ' (here ' $\mathrm{x}$ ' is the predecessor of ' $\mathrm{y}$ '). The rework process for an activity starts only when it receives confirmed parameters from all its predecessors.

Finally, there are some activities which are the end activities. These activities have predecessor(s) but no successor and denoted by subscript ' $k$ '. Design process for k-type activities is depicted by the right portion of the Figure which is similar to j-type activity except estimation part. Since these are the end activities, estimation is unnecessary to early release its parameter. Design process finishes with checking for rework. The following section elaborates how specific network can be generated automatically using the generalized network. 
Activity $\mathbf{A}^{\mathrm{i}}$

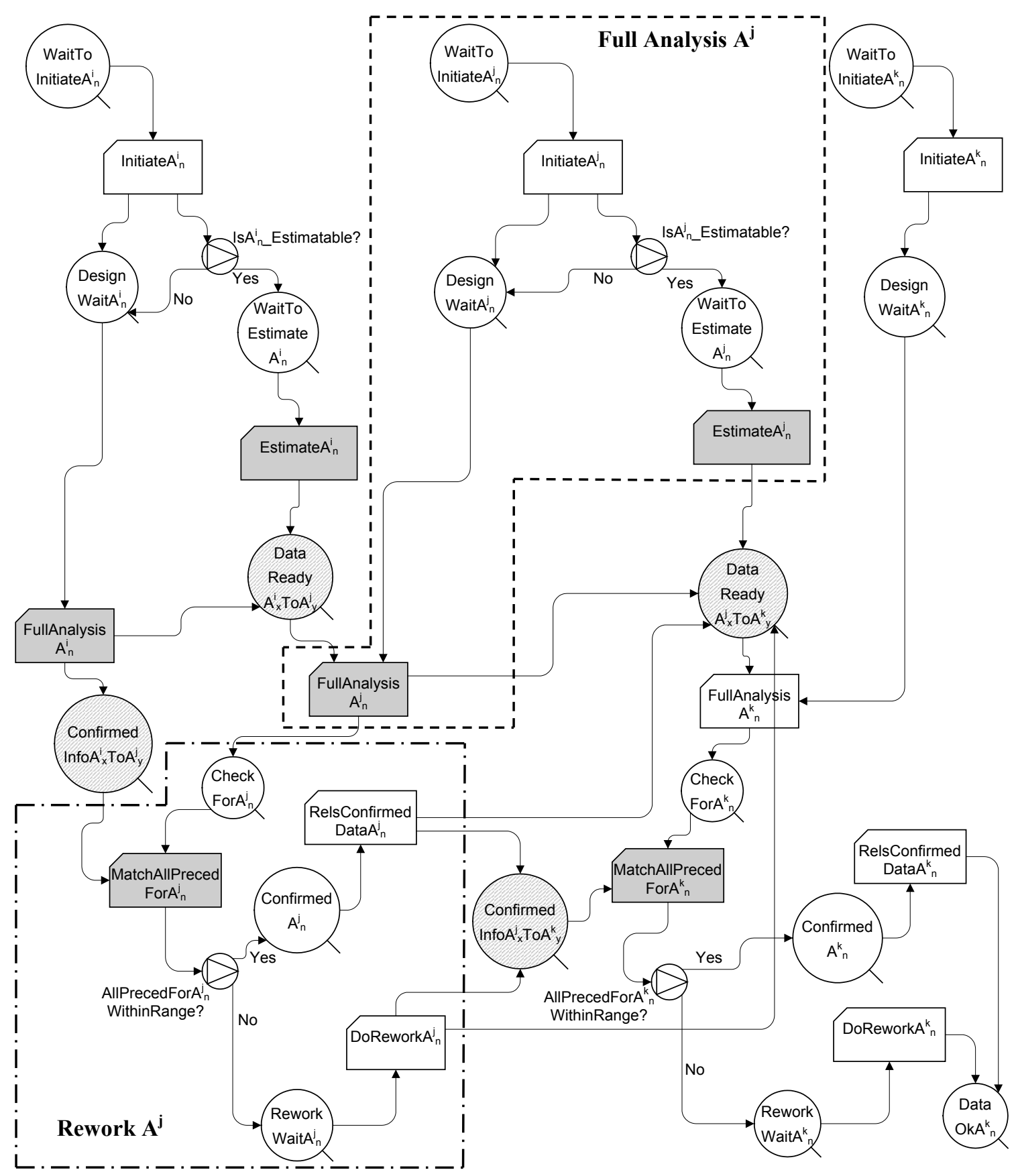

Figure 4: Generalized simulation network

\section{AUTOGENERATION OF THE SIMULATION NETWORK}

Abovementioned generalized network depicts the design processes for each type of activities with associated nodes and links. Among these nodes, some are common to all activities (such as the Combi nodes 'InitiateA' and 'FullAnalysisA'); some are specific to different type of activities (for instance, Fork node 'IsA_Estimable?' is belongs to ' $\mathrm{i}$ ' and ' $\mathrm{j}$ ' type activities); and some are based on predecessor and successor relationships (such as Queue node 'DataReady $A_{x} T o A_{y}$ ' ' if there is a dependency between activity $A_{x}$ to $A_{y}$ ). All the different types of nodes along with the links for the generalized network have been de- 


\section{Hossain and Chua}

fined once with subscript $x$ and $y$. To develop the network automatically, replications of nodes and links as needed for each type of activities as well as dependent Queues, have been done using 'IF' and 'WHILE' loop. The 'IF' blocks check for logical relationships where as the 'WHILE' blocks do a thorough search form the first activity to the last activity. Figure 5 shows two such examples of replication. In the first instance, following the DSM as depicted in Table 1, the coding in Figure 5(a) will generate six Forks: 'Is $A_{1_{-}}$Estimable?' through 'Is $A_{\sigma_{-}}$Estimable?' and not 'Is $A_{7_{-}}$Estimable?' since estimability is checked for ' $\mathrm{i}$ ' and ' $\mathrm{j}$ ' type of activities only in order to facilitate early information sharing and not for ' $\mathrm{k}$ ' type activities as $\mathrm{A}_{7}$.

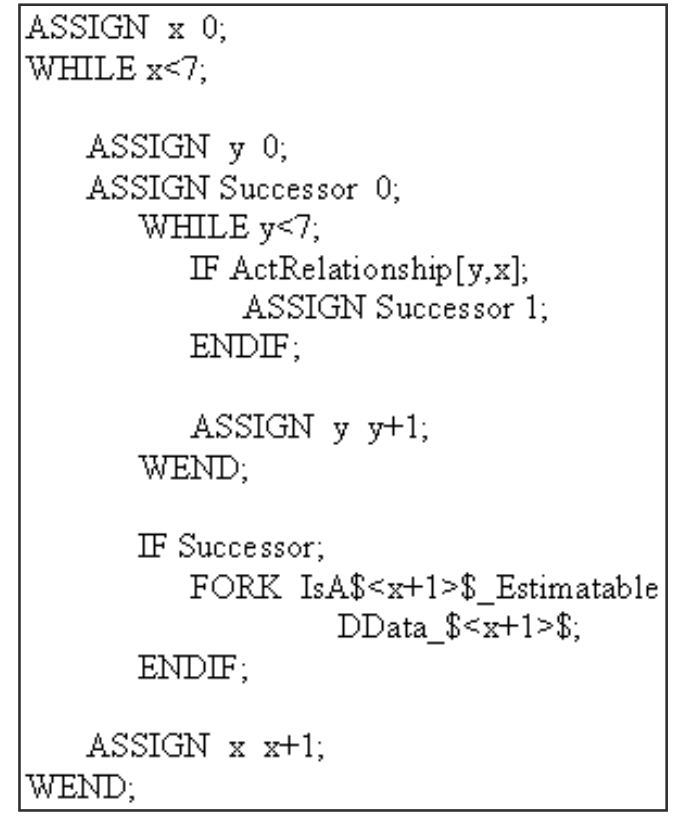

a)

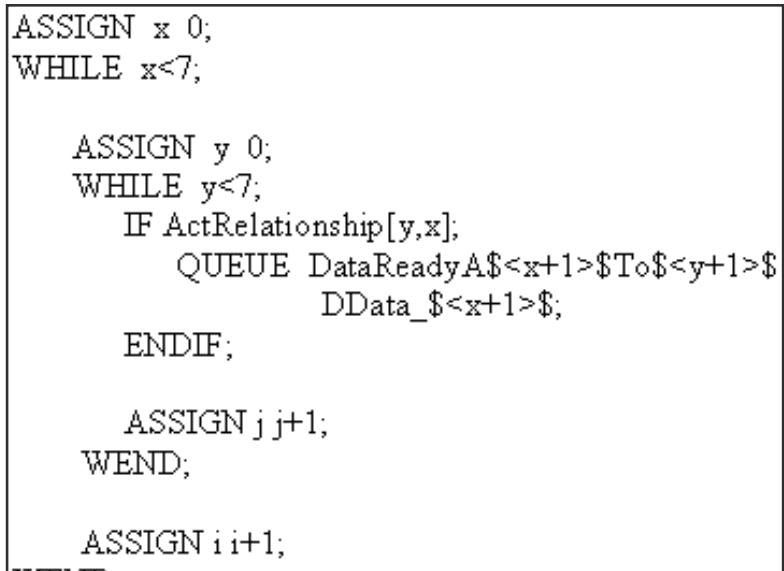

Figure 5: Autogeneration of the Fork 'Is $A_{x_{-}}$Estimable?' and the Queue 'DataReady $A_{x} T o A_{y}$ '

Similarly, Figure 5(b) will generate Queues 'DataReady $A_{x} T o A_{y}$ ' for the entire design project, whenever there is a dependency between activity $\mathrm{A}_{\mathrm{x}}$ and $\mathrm{A}_{\mathrm{y}}$. For example, activity $\mathrm{A}_{3}$ is dependent on $\mathrm{A}_{1}$ and $\mathrm{A}_{2}$; consequently, two Queues 'DataReady $A_{1} T o A_{3}$ ' and 'DataReady $A_{2} T o A_{3}$ ' will be generated to transmit parameter in the full analysis of $\mathrm{A}_{3}$ and so on.

In the similar manner, with the DSM as input, full network can be generated automatically for the entire project. Moreover, different attributes of the design activities such as duration for each activity, estimability, time to do estimation, time to do rework, and so on, are set with different arrays. These attributes are hooked with the generated network to run the simulation. Following section illustrate the effectiveness of the proposed model with a case study.

\section{CASE STUDY}

Effectiveness of the proposed model has been studied with a project in the oil and gas industry in Singapore. It is a design and build contract and the design master plan comprises 83 design activities with multi-disciplinary dependencies such as: process study, civil and structural, equipment, piping, instrumentation and electrical. The company is expected to finish the design phase by 12 months. With the traditional finish-start dependencies, the design project requires 432 working days to complete which far exceed the expected duration and this is typical of all design projects.

Chua and Hossain (2008) showed utilizing early estimated parameter can reduce overall design completion accounting the time required for rework. From the nature of the activities and based on inputs from Engineers, 40 activities (out of 83) are deemed to be estimable so that estimation can be done to obtain preliminary parameters' value. Time to do estimate is assumed to be $40 \%$ of the duration of the full analysis with a coefficient of variation of $20 \%$. To measure the effect of rework, it has been assumed that whenever an activity requires rework, time to do that is $20 \%$ of its original full analysis; however, any other duration can be assigned depending on the characteristics of the activity. To schedule the design project with these 40 estimable activities, a simulation network has to be developed with all the attributes of the design activities. Developing the simulation network as depicted in Figure 2 for the entire design project is very tedious and knowledge demanding. 


\section{Hossain and Chua}

To develop the specific network, the dependency relationships as provided by the Engineers in the project, are represented into a DSM. Defining the network in generalized way as described by Figure 4 and with the DSM as input, it is found to be effective to automatically generate the whole network.

With the abovementioned inputs, simulation is run for the generated network for one thousand $(1,000)$ times. The mean completion of the design project has been found to be 303 working days with a standard deviation of 23.6, though it requires 80 mandays of rework. If the estimated parameters are assumed to be accurate so that rework is unnecessary, design completion reduced to 300 days. So, 80 mandays of rework is lengthening the project duration only by three (3) days, since most of the reworks can be done parallel along with other design activities. The simulation model also provides a probabilistic schedule with average start and end dates of all the events with their standard deviations.

The simulation network has been studied in further details to better elaborate the usefulness of the concept of autogeneration. As noticed earlier, the design project requires 432 working days with traditional finish-start relationships where no early information is incorporated. The simulation yields the same result if estimability for all activities is set as ' 0 ' in the generalized network. In this case, no activity is utilizing early estimated information and waiting for full analysis so that rework is unnecessary. This scenario gives the traditional finish-start schedule. While, if all the activities are assumed to be estimable, then simulation result depicts that the project requires a mean of 190 working days with a total 146 mandays of rework. Similarly, other attributes of the design activity can be changed based on the characteristics of the activity. So, the network can be generated automatically based on the dependency relationships (DSM) and with design attributes without redrawing the simulation network for each design project. After that, simulation can be run to study the project behavior easily.

\section{CONCLUSION}

This study proposes a technique to generate simulation network automatically for any design process based on the DSM. The generated simulation network is integrated with the given attributes (estimability, time to do estimation, time to do full analysis and so on) of design activities in order to study the project behavior. Simulation can be run for the generated network to get a probabilistic schedule with reduced duration. Concept of estimability and rework has been utilized in an attempt to reduce the overall project completion time. The case study presented from a real life project describes the effectiveness of the model. Simulation can be run for any design project by changing the input arrays. This will save the tedious job of developing simulation model for individual project and is also useful for those who have relatively little knowledge on simulation technique.

The current study has not considered the resource constraint in scheduling the design process. Any resources such as design specialists or other physical resources for which design activities are delayed can easily be incorporated with the current model. All the resources and manpower can be stored in different pools (Queues) and can be used through different links for different activities. Queues and links can be defined in generalized way and can be used for autogeneration for different resources needed by different activities. After that, optimum amount of resources and their efficiencies can be measured through simulation.

\section{REFERENCES}

Austin, A., A. Baldwin, B. Li, and P. Waskett. 2000. Analytical Design Planning Technique (ADePT): a Dependency Structure Matrix Tool to Schedule the Building Design Process. Construction Management and Economics 18: 173-182.

Chua, D.K.H, and G.M. Li. 2001. Modeling Construction Operation with RISim. Computing in Civil Engineering 5-4: 320328.

Chua, D. K. H, and G. M. Li. 2002. RISim: Resource-Interacted Simulation Modeling in Construction. Construction Engineering and Management 128-3: 195-202.

Chua, D. K. H., and M. A. Hossain. 2008. Reduced duration of the design projects with the concept of early estimation of design tasks. In Proceeding of $16^{\text {Th }}$ Annual Conference of the IGLC, ed. P. Tzortzopoulos, and M. Kagioglou, 485-495. Manchester, U.K.

Halpin, D. W., and R. W. Woodhead. 1976. Design of Construction and Process Operations. John Wiley and Sons, New York.

Ioannou, P. G. 1990. UM-CYCLONE Discrete Event Simulation System User's Guide. UMCEE Report No. 89-12, Civil and Environmental Engrg Dept., University of Michigan, Ann Arbor, Michigan.

Ioannou, P. G., and J. C. Martinez. 1996. Scalable Simulation Models for Construction operations. In Proceeding of 1996 Winter Simulation Conference, ed.J. M. Charnes, D. J. Morrice, D. T. Brunner, and J. J. Swain. 1321-1328. Society for Computer Simulation, San Diego, Calif.

Kim, K. J., and G. E. Gibson Jr. 2003. Interactive simulation modeling for heavy construction operations. Automation in Construction 12: 97-109. 


\section{Hossain and Chua}

Lee, D. E., and D. Arditi. 2006. Automated Statistical Analysis of Stochastic Project Scheduling Simulation. Construction Engineering and Management 132-3: 268-277.

Liu, L. Y., and P. G. Ioannou. 1992. Graphical Object-Oriented Discrete-Event Simulation System" In Proceeding of the 1992 Winter Simulation Conference, ed. J. J. Swain, D. Goldsman, R. C. Crain, and J. R. Wilson, 1285-1291. Arlington, Virginia.

Martinez, J. C. 1996. STROBOSCOPE State and Resource Based Simulation of Construction Processes. Ph.D. Thesis, Civil \& Envir. Engrg., Univ. of Michigan, Ann Arbor, Michigan. Available via <http://www.cem.umich.edu/Ioannou/StrobWeb/Stroboscope/StrbPubs/UM96_Thesis/UMTh esis.pdf> [accessed April6, 2009].

Odeh, A. M. 1992. CIPROS: Knowledge-Based Construction Integrated Project and Process Planning Simulation System. PhD Dissertation, University of Michigan, Ann Arbor, Michigan.

Steward, D. V. 1981. System Analysis and Management: Structure, Strategy and Design. Petrocelli Book, McGraw Hill, New York.

\section{AUTHOR BIOGRAPHIES}

M. A. HOSSAIN is pursuing his Ph.D. degree in the Civil Engineering Department at the National University of Singapore. He obtained B.Eng. degree (2005) from Bangladesh University of Engineering and Technology. His research area includes physics of design flow in construction industry and simulation-based scheduling of design and construction projects. His email is <aslamenus.edu.sg >.

D. K. H. CHUA is an Associate Professor in the Civil Engineering department at the National University of Singapore. He received his Ph.D. in the Civil and Environmental Department at the University of California, Berkeley in 1989 and an MSc in 1986 from the same University. He also obtained his MEng from National University of Singapore in 1982 and graduated from Adelaide University with BEng in 1980. His research interest includes Lean construction, simulation, 4D CAD, construction safety, and IT in construction. His email and web addresses are <cvedavidenus.edu.sg> and <http://www.eng.nus.edu.sg/civil/people/cvedavid/david.html>. 